\title{
NEW EXACT ITERATIVE METHOD FOR THE CAPACITATED VEHICLE ROUTING PROBLEM
}

The aim of the Capacitated Vehicle Routing Problem is to find a set of minimum total cost routes for a fleet of capacitated vehicles, to serve a set of customers. This problem belongs into category of NP hard problems that can be exactly solved only for small instances. In this paper we propose an exact iterative method for the CVRP based on the solution of Mixed Linear Programming model, which is able to solve problem for relatively larger instances in acceptable computing time.

Keywords: Capacitated vehicle routing problem, mixed linear programming model.

\section{Introduction and problem description}

The Capacitated Vehicle Routing Problem (CVRP) is one of fundamental problems in combinatorial optimization with a number of practical applications in transportation, distribution and logistics. The aim of CVRP is to find a set of minimum total cost routes for a fleet of capacitated vehicles, based at one depot, to serve a set of customers under the following constraints:

(1) each route begins and ends at the depot,

(2) each customer is visited exactly once,

(3) the total demand of each route does not exceed the capacity of the vehicle [1].

Figure 1 shows an example of a feasible CVRP solution with seven customers and equal capacity $Q=50$ of vehicles.

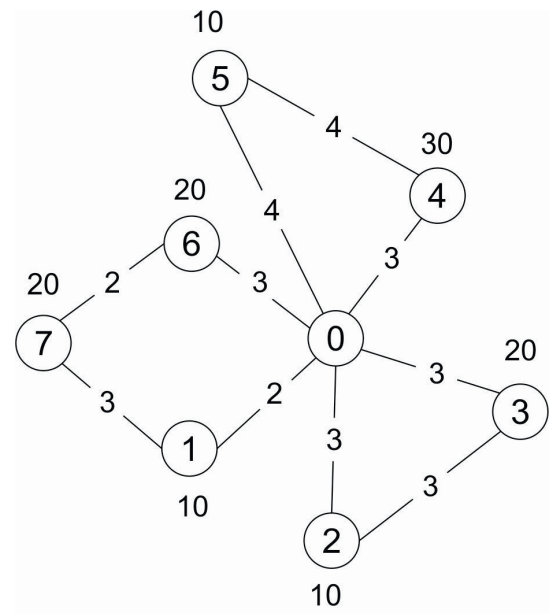

Fig. 1 A feasible CVRP solution, $n=7, Q=50$

\section{Mathematical formulation}

Let $G=(V, H)$ be a complete directed graph with $V=\{0,1,2, \ldots, n\}$ as the set of nodes and $H=\{(i, j): i, j \in V, i \neq j\}$ as the set of arcs, where node 0 represents the depot for a fleet of identical vehicles of capacity $Q$ and remaining $n$ nodes represent geographically dispersed customers. Each customer $i \in V-\{0\}$ has a certain positive demand $d_{i} \leq Q$ and $d_{0}=0$. Non negative travel cost $c_{i j}$ is associated with each $\operatorname{arc}(i, j) \in H$. The cost matrix is symmetric, i.e. $c_{i j}=c_{i j}$ for all $i, j \in V, i \neq j$ and satisfies the triangular inequality, $c_{i j}+c_{j k} \leq c_{i k}$ for all $i, j, k \in V$ [2]. The minimum number of vehicles needed to serve all customers is $p=\left\lceil\sum_{i=1}^{n} d_{i} / Q\right\rceil$.

Two-index decision variables $x_{i j}$ are used as binary variables equal to 1 if $\operatorname{arc}(i, j)$ belongs to optimal solution and 0 otherwise. For all pairs of nodes $i, j, i \neq j$ we calculate the savings $s_{i j}$ for joining the cycles $0 \rightarrow i \rightarrow 0$ and $0 \rightarrow j \rightarrow 0$ using arc $(i, j)$ :

$s_{i j}=c_{i 0}+c_{0 j}-c_{i j}$

as in Clarke and Wright's saving method [3]. The saving $s_{i j}$ is illustrated in Fig. 2. In the left part, customers $i$ and $j$ are served by their own vehicle, in the right part, customers $i$ and $j$ are served by one vehicle.

Now, instead of minimizing the total cost, we can maximize the total saving. To ensure continuity of route and to elimininate subtours we define an auxiliary continuous variable $y_{i}, d_{i} \leq y_{i} \leq Q$ for $i \in\{1,2, \ldots, \mathrm{n}\}, y_{0}=0$, which shows (in the case of collection of the goods) the vehicle load after visiting customer $i$ [4]. To make modeling easier, each feasible route $0 \rightarrow v_{1} \rightarrow v_{2} \rightarrow \ldots \rightarrow v_{\mathrm{k}} \rightarrow 0$ we

\footnotetext{
* Zuzana Borcinova, Stefan Pesko

Department of Mathematical Methods and Operations Research, Faculty of Management Science and Informatics, University of Zilina, Slovakia E-mail: zuzana.borcinova@fri.uniza.sk
} 

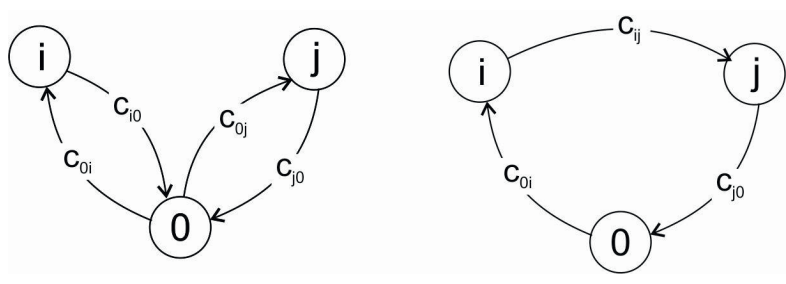

Fig. 2 Saving $s_{i j}$ for customers $i$ and $j$ in cycle $0 \rightarrow i \rightarrow j \rightarrow 0$

replace by path from node 0 to node $v_{k}$, i.e. $0 \rightarrow v_{1} \rightarrow v_{2} \rightarrow \ldots \rightarrow v_{\mathrm{k}}$. For example, a feasible solution is illustrated in Fig. 3. Two values are assigned to each node $i$ - the demand $d_{i}$ of customer $i$ and the load $y_{i}$ of vehicle after visiting customer $i$ (inside the brackets). The path $0 \rightarrow 2 \rightarrow 3$ represents the route $0 \rightarrow 2 \rightarrow 3 \rightarrow 0$, where customer 2 has demand $d_{2}=10$, customer 3 has demand $d_{3}=20$, the value of the vehicle load in node 2 is $y_{2}=10$ and in node 3 is $y_{3}=30$.

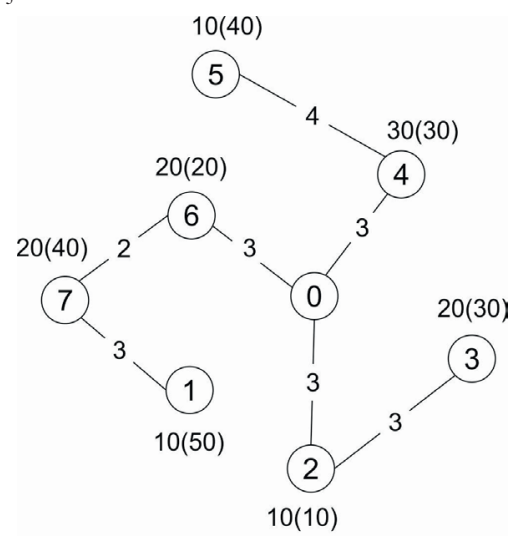

Fig. $3 n=7, Q=50$

The CVRP can be formulated as following mixed linear programming model (CVRP 1):

Maximize $\sum_{i=1}^{n} \sum_{j=1, j \neq i}^{n} s_{i j} x_{i j}$

Subject to $\sum_{j=1}^{n} x_{0 j}=p$

$\forall j \in\{1,2, \ldots, n\}$

$\sum_{i=0, i \neq j}^{n} x_{i j}=1$

$\forall j \in\{1,2, \ldots, n\}$

$\sum_{i=0, i \neq j}^{n} x_{i j} \leq 1$

$\forall j \in\{1,2, \ldots, n\}$

$y_{i}+d_{j} x_{i j}-Q\left(1-x_{i j}\right) \leq y_{j} \forall j \in\{1,2, \ldots, n\}, i \neq j$

$d_{i} \leq y_{i} \leq Q$

$\forall j \in\{1,2, \ldots, n\}$

$x_{i j} \in\{0,1\} \quad \forall(i, j) \in H$

In this formulation, the objective function (1) maximizes the total travel saving. The constraints (2) impose that exactly $p$ vehicles leave the depot, (3) and (4) are indegree and outdegree constraints for customers. Constraints (5) are route continuity and subtour elimination constraints, ensuring that the solution contains no subtour disconnected from the depot and that the vehicle load is non decreasing step function in accordance with the demand of the customers which are on the route of the vehicle. Constraints given in (6) are capacity bounding constraints and restrict the upper and lower bounds of $y_{i}$.

The Capacitated Vehicle Routing Problem belongs into category of NP hard problems that can be exactly solved only for small instances of the problem. We propose an exact iterative method for the CVRP based on the solution of Mixed Linear Programming model, which is able to solve problem for relatively larger instances in acceptable computing time.

\section{Exact iterative method}

The essence of our method is the iterative improvement of initial feasible solution $S$ by replacement of some of the arcs with the other ones, obtained by exact solution of the simpler problem.

Algorithm:

Step 1: Apply a fast heuristic method to find an initial (feasible) CVRP solution $S_{0}$. Set $S=S_{0}$.

Step 2: Create a list $E$ of arcs which belong to solution $S$ and are not incident with the depot. Let $m=|E|$

Step 3: Set values $k=1, m_{1}=m-\delta$ and $m_{2}=m-1$, where $\delta$ is a predetermined integer value $1<\delta<m$.

Step 4: Find an optimal solution $S_{k}$, so that the set $E_{k}$ of arcs which belong to solution $S_{k}$ and are not incident with the depot contains minimally $m_{1}$ and maximally $m_{2}$ of arcs from $E$, i.e. we add to (CVRP l) the constraints:

$$
\sum_{(i, j) \in E}^{n} x_{i j}=z
$$

$m_{1} \leq z \leq m_{2}$

where integer variable $z$ determines how many arcs from $E$ are retained in $E_{k}$.

Step 5: If the solution $S_{k}$ is better than the solution $S$, then set $S=S_{k}$ and continue to Step 2 .

Else set values $k=k+1, m_{1}=m_{1}-\delta$ and $m_{2}=m_{2}-\delta$ and go to Step 4.

The algorithm can be stopped after a predetermined computational time or number of iterations without improvement.

\section{Computational experiments}

Our improved model was coded in Python 3.4 [5] and solved using Gurobi 6.5 [6] on PC with Intel Xeon 32 cores, $2.4 \mathrm{GHz}$, 256 GB RAM. We have done our experiments on 7 instances 
taken from classical sets of CVRP benchmark from Augerat et al. (1995). The input data is available online at [7].

For finding the starting solution we applied the well known heuristic proposed by Clarke and Wright (1964). Each instance data was solved to optimality for various values of parameter $\delta$. In Table 1 the corresponding computational times are shown.

Results of computational experiments with parameter $\delta \quad$ Table 1

\begin{tabular}{lrrrrrrrrr} 
& & & & & & \multicolumn{4}{c}{ time (sec.) } \\
\cline { 8 - 10 } Instance & $\mathrm{n}$ & $\mathrm{Q}$ & $\mathrm{p}$ & Start & Opt. & $\delta=2$ & $\delta=3$ & $\delta=4$ \\
\hline A-n32-k5 & 31 & 100 & 5 & 901 & 784 & 9 & $\mathbf{6}$ & 7 \\
A-n34-k5 & 32 & 100 & 5 & 886 & 778 & 335 & 136 & $\mathbf{5 0}$ \\
A-n36-k5 & 35 & 100 & 5 & 870 & 799 & 12048 & 3458 & $\mathbf{1 8 4 7}$ \\
A-n38-k5 & 37 & 100 & 5 & 828 & 730 & 81 & $\mathbf{7 6}$ & 134 \\
A-n44-k6 & 43 & 100 & 6 & 1100 & 937 & 718 & 748 & $\mathbf{3 0 2}$ \\
A-n53-k7 & 52 & 100 & 7 & 1167 & 1010 & $\mathbf{1 1 6 5}$ & 5608 & 18134 \\
A-n55-k9 & 54 & 100 & 9 & 1291 & 1073 & 37884 & $\mathbf{4 9 6 4}$ & 165540
\end{tabular}

$n$ - number of the customers, $Q$ - capacity of the vehicles, $p$ - number of the vehicles, Start - cost of the initial solution, Opt - cost of the optimal solution from literature

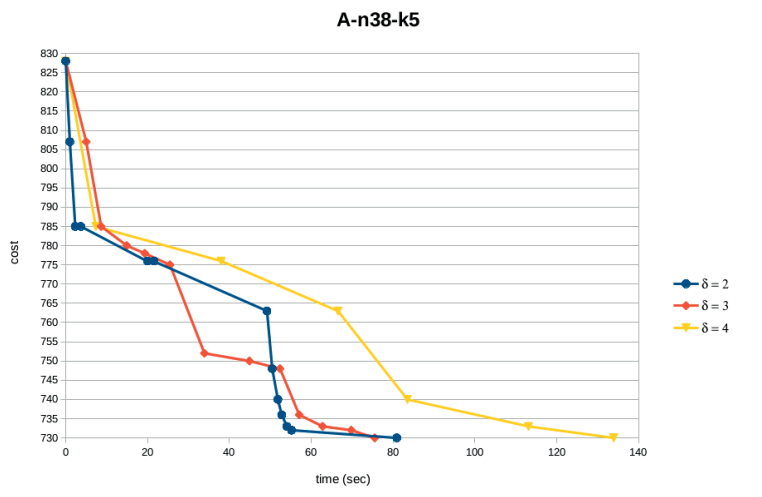

Fig. 4 Time evolution of solution improvement for various $\delta$
Figure 4 illustrates time evolution of solution improvement for A-n 38-k5 instance with 37 customers and 5 vehicles, from initial cost 828 to optimal cost 730 .

\section{Conclusions}

We have proposed an exact iterative method for the CVRP based on the solution of Mixed Linear Programming model. From our computational experiments is evident that it is not possible to determine which value of parameter delta is the most appropriate for given instance. This led us to the idea of parallel computation with respect to the parameter delta. Our method seems to be promissing, because it is able to exactly solve problem for relatively large instance and we plan to develop it in the further research.

\section{Acknowledgment}

The research of Z. Borcinova was supported by the Scientific Grant Agency of the Ministry of Education of the Slovak Republic and the Slovak Academy of Sciences under project VEGA 1/0518/15 "Resilient rescue systems with uncertain accessibility of service", and the research of S. Peško was supported by the Slovak Research and Development Agency under projects APVV-0760-11 "Designing of fair service systems on Transportation networks" and APVV-14-0658 "Optimization of urban and regional public personal transport".

\section{References}

[1] LAPORTE, G.: What You Should Know about the Vehicle Routing Problem, Naval Research Logistics, 54: 811-819, 2007.

[2] TOTH, P., VIGO, D.: Models, Relaxations and Exact Approaches tor Capacitated Vehicle Routing Problem, Discrete Applied Mathematics, 123: 487-512, 2002.

[3] LAPORTE, G.: The Vehicle Routing Problem: An Overview of Exact dnd Approximate Algorithms, European J. of Operational Research 59: 345-358, 1992.

[4] KARA, I.: Tightening Bounding Constraints of the Miller-Tucker-Zemlin Based Formulation of the Capacitated Vehicle Routing Problems and Some Extensions, Proc. of the $2^{\text {nd }}$ intern. conference on Manufacturing Engineering, Quality and Production Systems, edited by C. Panait et al., WSEAS Press, Constantza, 2010, pp.137-142.

[5] The Python Language Reference, 1990 - 2016, Python Software Foundation, http://docs.python.org/3/.

[6] Gurobi Optimizer Reference Manual, version 6.5, 2016, Gurobi Optimization, Inc., http://www.gurobi.com.

[7] Branch Cut and Price Resource Web, Vehicle Routing Data Sets USA, http://www.coin-or.org/SYMPHONY/branchandcut/VRP/ data/. 